\title{
Detecção Automática da Onda R do ECG por Limiar e Transformada de Wavelet Discreta: Um Comparativo do Cálculo da Frequência Cardíaca
}

\author{
Maikon L. Santos*. Guilherme F. Faria** \\ Vínícius R. Cota* \\ *Laboratório Interdisciplinar de Neuroengenharia e Neurociência-LINNce \\ Departamento de Engenharia Elétrica UFSJ - DEPEL \\ Universidade Federal de São João del-Rei - UFSJ \\ Pça Frei Orlando, 170, Centro, São João del-Rei-MG, Brasil. CEP: 36307-352 \\ E-mails: maikon.lorran@hotmail.com,vrcota@ufsj.edu.br \\ Tel.: 32-3379-2552 \\ **Laboratório de Sistemas Dinâmicos - LASID \\ Departamento de Engenharia Mecânica UFSJ - DEMEC \\ Universidade Federal de São João del-Rei - UFSJ \\ Pça Frei Orlando, 170, Centro, São João del-Rei-MG, Brasil. CEP: 36307-352 \\ E-mail: guilhermefonsecafaria@gmail.com \\ Tel.: 32-3379-2595
}

\begin{abstract}
During the last decades, several methods have been developed for the automatic detection of electrocardiographic (ECG) signal characteristics, based on mathematical tools. Some of them are the Continuous Wavelet Transform (CWT), the Discrete Wavelet Transform (DWT), Principal Component Analysis (PCA), Hilbert Transform (HT), among many others. The transforms are applied in the algorithm, which is based on an adaptive threshold, in order to highlight only the R peaks of the ECG, so that the heart rate is calculated. The work carried out filtration and identification of the $\mathrm{R}$ peak for two different people. The first one, through the threshold detection, where the other P, Q, S, T and U waves were ignored, that is, they were submitted to a zero condition. After the best R-peak condition was found, heart rate was calculated. In the second person, we used the Wavelet Transform, which separates the frequency bands. It is possible to obtain only the frequency referring to the peak $\mathrm{R}$ and thus to calculate the heart rate. The ECG databases were obtained from the Massachusetts Institute of Technology (MIT). The results show the feasibility and efficacy of the methodology.
\end{abstract}

Resumo: Durante as últimas décadas foram desenvolvidos diversos métodos para a detecção automática das características dos sinais de eletrocardiogramas (ECG), baseados em ferramentas matemáticas. Algumas delas são: a Transformada Wavelet Contínua (TWC), a Transformada Wavelet Discreta (TWD), a Análise de Componentes Principais (ACP), a Transformada de Hilbert (TH), entre muitas outras. As transformadas são aplicadas no algoritmo, que é baseado em um limiar adaptativo com o objetivo de realçar apenas os picos R do ECG, para que a frequência cardíaca seja calculada. $\mathrm{O}$ trabalho realizou filtragem e identificação do pico R para duas pessoas diferentes. Sendo a primeira por meio da detecção de limiar, onde as demais ondas P, Q, S, T e U foram ignoradas, ou seja, submetidas a condição igual a zero. Depois de encontrada a melhor condição do pico R, a frequência cardíaca foi calculada. Na segunda pessoa foi utilizado a Transformada de Wavelet, que separa as bandas de frequências, sendo possível obter apenas a frequência referente ao pico R e assim, fazer o cálculo da frequência cardíaca. Os bancos de dados do ECG foram obtidos do Instituto de Tecnologia de Massachusetts (MIT). Os resultados obtidos mostram a viabilidade e eficácia da metodologia.

Keywords: Electrocardiogram; Wavelet Transform; Pattern Recognition; Systems Identification; Heart Rate; Heart Cycle; ECG; Electrophysiology of the Heart.

Palavras-chaves: Eletrocardiograma; Transformada de Wavelet; Reconhecimento de Padrões; Identificação de Sistemas; Frequência Cardíaca; Ciclo ardíaco; ECG; Eletrofisiologia do Coração.

\section{INTRODUÇÃO}

Com o avanço do processamento computacional, tem-se desenvolvido diversas técnicas de análise digital de sinais de eletrocardiograma (ECG). O eletrocardiograma é um exame médico que pode identificar diversos tipos de doenças cardíacas, sendo este de baixo custo e não invasivo (Silva et al., 2015). 
Segundo a Organização Mundial de Saúde (OMS), cerca de 15 $\%$ das causas de morte no mundo estão relacionadas a problemas cardiovasculares (Kohler et al., 2002). Nas últimas décadas diversas soluções/ferramentas têm sido propostas para o rápido diagnóstico de problemas cardíacos, baseando-se na análise do eletrocardiograma. O eletrocardiograma é o registro do potencial elétrico gerado pela atividade elétrica do coração (Madeiro et al., 2009). O sinal ECG é composto pela sucessão repetitiva de 5 ondas, onde cada uma destas representa uma parte do ciclo cardíaco. Essas são as ondas P, Q, R, S e T, cada uma possui uma faixa pré-determinada de amplitude e duração. Elas constituem um determinado comportamento das células que compõem o coração, mais especificamente, os processos de despolarização e repolarização do átrio e do ventrículo (Kohler et al., 2002).

A onda $\mathrm{P}$ representa a despolarização atrial, contudo a repolarização não é facilmente observada, devido ao fato de ocorrer durante a despolarização do ventrículo, que apresenta uma amplitude maior, cerca de $1 \mathrm{mV}$, quando comparada à amplitude produzida pela repolarização do átrio. A despolarização do ventrículo origina as ondas $\mathrm{Q}, \mathrm{R}$ e $\mathrm{S}$, estabelecendo o chamado Complexo QRS. A repolarização do ventrículo é dada pela onda T. A onda U, nem sempre presente no ECG, representa a repolarização da musculatura papilar e apresenta, normalmente, a mesma polaridade da onda $\mathrm{T}$, porém com uma amplitude inferior (Friesen et al., 1990).

Diversas técnicas de processamentos de sinais têm sido utilizadas nos últimos anos na análise de sinais do ECG. Entre elas destacam-se a Transformada Wavelet Discreta (TWD) e a Transformada Rápida de Fourier (FFT) e sua inversa, que são os dois métodos principais apresentados neste trabalho.

A Transformada Wavelet (TW) é um método matemático relativamente novo, que vem sendo estudado nos últimos anos para à análise de sinais. Sua fundamentação tem como base no trabalho de Fourier, que no século XVIII demonstrou a importância dessa análise no domínio da frequência (Silva et al., 2015). A TW efetua a análise em tempo-frequência do sinal, diferentemente de outros métodos utilizados. A TWD pode efetuar a separação do sinal em diversas componentes, o que permite analisar cada parte individualmente. Dessa forma, as frequências referentes a onda $\mathrm{R}$ (picos $\mathrm{R}$ ) podem ser isoladas e analisadas. $\mathrm{Na}$ detecção por limiar, filtros separam as ondas e frequências pelo valor de amplitude, sendo ignoradas ou classificadas como possíveis ondas de pico R sem a presença de artefatos. Uma função disponibilizada no site Mathworks é a responsável pela filtragem dos picos e um laço realiza a classificação. Essa função é amplamente utilizada por pesquisadores em todo o mundo para tratamento do sinal de ECG.

No tema de deteç̧ão computacional de sinais com ECG baseado na Transformada Wavelet, é possível encontrar vários trabalhos publicados na literatura técnica-científica. Dentre os mais recentes: (Junior et al., 2016; Gopalakrishnan et a., 2013; Merah et al., 2015).

Portanto, este trabalho propõe a aplicação de dois métodos diferentes para filtragem e processamento do sinal do ECG.

\section{METODOLOGIA}

\subsection{Filtragem por limiar e normalização}

A filtragem por limiar e sua classificação tem seu algoritmo descrito em seis etapas:

1. Retirada dos sinais iniciais e finais do ECG. Os 85 valores em ambas as extremidades são desconsiderados por conter excesso de ruído.

2. Retirada das componentes de baixa frequência pela FFT e retornado para o tempo pela FFT inversa. Este é considerado o filtro inicial do tratamento do ECG.

3. O segundo filtro é realizado pela função ecgdemowinmax, disponibilizado no site Mathworks. O filtro por limiar retira do ECG as ondas, P, Q, S, T e quando necessário a onda U. Por se tratarem de ondas com amplitude mais baixas em relação a $\mathrm{R}$, o filtro consegue classificar com certa facilidade, a onda $\mathrm{R}$ a cada batimento cardíaco. A Figura 1 mostra todas as ondas citadas anteriormente, sendo que a onda $\mathrm{R}$ pode chegar a $1 \mathrm{mV}$.

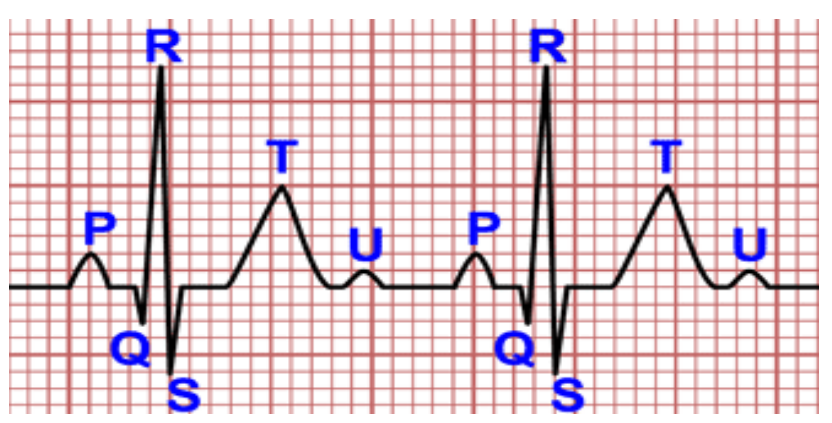

Fig. 1 Ondas do ciclo cardíaco.

4. Após as duas primeiras filtragens, os picos são detectados. Assim, podendo verificar se ainda há ou não componentes que podem ser pico de onda R. Além dessa classificação, o algoritmo já normaliza os dados para facilitar à análise.

5. Se após as filtragens iniciais ainda houver ondas que não são caracterizadas como pico R (falso positivo), um terceiro filtro é realizado no intuito de retirar tais ondas. A mesma função ecgdemowinmax recebe $\mathrm{o}$ incremento necessário para aperfeiçoar o código, mudando apenas os valores considerados inicialmente. Caso o resultado seja satisfatório no primeiro e segundo filtros, o terceiro pode ser ignorado, pois, pode-se gerar resultados ambíguos e ter um gasto computacional desnecessário.

6. Nesta etapa, o algoritmo apresenta os resultados obtidos graficamente, o valor médio e o valor ponto a ponto da frequência cardíaca. Para esses cálculos o algoritmo também retorna todos os pontos de pico $\mathrm{R}$ encontrados no ECG original. Também são apresentados os resultados que foram considerados no primeiro e segundo filtro e desconsiderados no terceiro.

\subsection{Filtro por Transformada de Wavelet Discreta}


O sinal analisado pela TWD é dividido em duas componentes: as aproximações que são componentes de alta escala e baixa frequência, e os detalhes que são componentes de baixa escala e alta frequência, conforme ilustrado na Figura 2.

A TWD realiza à multiresolução de um determinado sinal, tradicionalmente por meio de um algoritmo iterativo. Assim, a decomposição do sinal original é realizada em sub-bandas, que correspondem às componentes do sinal original em diferentes faixas de frequências. $O$ processo de filtragem realiza a decomposição em baixa resolução do sinal original, denominada de aproximação do sinal e em uma versão que contém basicamente os detalhes do sinal original. A filtragem é seguida pelo processo de subamostragem, ambas figurando como um estágio completo da decomposição.

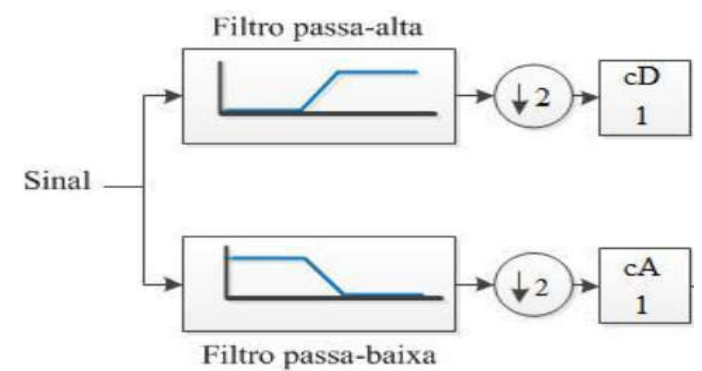

Fig. 2 Filtragem e separação do sinal por filtros passa-alta e passa baixa.

Essa segunda decomposição é efetuada sobre a primeira aproximação gerada, e assim sucessivamente. Este processo é chamado de árvore de decomposição Wavelet ou encadeamento, como apresentado na Figura 3. A cada encadeamento a frequência de amostragem é dividida por 2.

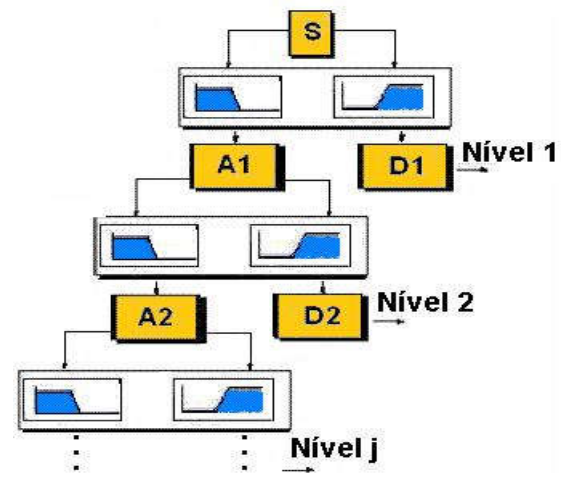

Fig. 3 Árvore ou encadeamento da Decomposição de Wavelet.

A Transformada de Wavelet usa uma família de funções de análise, as quais são utilizadas para representar o sinal em diferentes resoluções, que por sua vez, informam o conteúdo de diferentes bandas de frequência (Madeiro et al., 2009). A família de funções $\Psi a$,b é deduzida a partir da Wavelet-mãe $\Psi(\mathrm{t})$, dada pelas equações 1 e 2 .

$$
\begin{gathered}
T W^{f}(a, b)=\int_{-\infty}^{\infty} f(t) \Psi_{a, b}(t) d t \\
T W^{f}(j, k)=a_{0}^{-j / 2} \int_{-\infty}^{\infty} f(t) \Psi\left(\frac{t-b}{a}\right) d t
\end{gathered}
$$

Sendo o escalonamento representado pela letra $a$ e o deslocamento pela letra $b$. As equações 1 e 2 tratam da Transformada de Wavelet do tempo contínuo, mas são base para a equação no tempo discreto, conforme mostrado na equação 3 .

$$
T W^{f}(j, k)=a_{0}{ }^{-j / 2} \int_{-\infty}^{\infty} f(t) \Psi\left(a_{0}^{-j} t-K b_{0}\right) d t
$$

Onde: $\mathrm{j}, \mathrm{k} \in \mathrm{Z}$

$$
\begin{aligned}
& a=a_{0}^{j} \\
& b=b_{0} \cdot a_{0}^{j}
\end{aligned}
$$

Assim, há uma correspondência entre escalas e frequências das Wavelets, isto é:

- Para baixos $a \Rightarrow$ Wavelets curtas $\Rightarrow$ rápidas mudanças $\Rightarrow$ altas frequências $W$.

- Para altos $a \Rightarrow$ Wavelets longas $\Rightarrow$ mudanças lentas $\Rightarrow$ baixas frequências $W$.

$\mathrm{Na}$ Tabela 1, são mostra as faixas de frequência para cada escala Wavelet.

Tabela 1. Faixa de frequência da escala Wavelet

\begin{tabular}{cc}
\hline Coeficientes & Faixa de Frequência $\mathbf{( H z )}$ \\
\hline d1[n] & $90-180$ \\
\hline d2[n] & $45-90$ \\
\hline $\mathbf{d 3}[\mathbf{n}]$ & $22,5-45$ \\
\hline $\mathbf{d} 4[\mathbf{n}]$ & $11,5-22,5$ \\
\hline $\mathbf{d 5}[\mathbf{n}]$ & $5,625-11,25$ \\
\hline $\mathbf{d 6}[\mathbf{n}]$ & $2,8125-5,625$ \\
\hline $\mathbf{d} 7[\mathbf{n}]$ & $1,4062-2,8125$ \\
\hline $\mathbf{d 8}[\mathbf{n}]$ & $0,7031-1,4062$ \\
\hline $\mathbf{d} 9[\mathbf{n}]$ & $0,3115-0,7031$ \\
\hline $\mathbf{d} 10[\mathbf{n}]$ & $0,1757-0,3515$ \\
\hline
\end{tabular}

Pode-se observar com base na Tabela 1, que grande parte da energia do complexo QRS está distribuída entre as escalas 3, 4, 5 e 6, uma vez que essa parcela de energia está situada na faixa de $3 \mathrm{~Hz}-40 \mathrm{~Hz}$. Contudo, é possível detectar a presença do complexo nas demais escalas. Já as energias das ondas $\mathrm{P}$ e T estão praticamente situadas na faixa de $3 \mathrm{~Hz}-10 \mathrm{~Hz}$, que são as escalas 5 e 6 (Thakor et al., 1990).

Então, tendo em vista os valores das bandas de energia dos coeficientes de Wavelet, é possível estudar o comportamento no domínio do tempo, das componentes do sinal ECG em diversas escalas de frequência. Sendo assim, uma das grandes vantagens da Transformada Wavelet: análise em tempo e frequência. Outra vantagem da utilização da TW é a filtragem de ruídos de alta frequência que estão contidos nos sinais ECG, além de outros ruídos, amenizando o efeito destes sobre a análise do sinal. Os coeficientes de aproximação são resultados de filtros passa-baixas e os coeficientes Wavelet de filtros passa-altas (Junior et al., 2016). A detecção do pico R é feita com base nos coeficientes Wavelet, utilizando métodos threshold, ou seja, baseando-se em valores limiares para a detecção. 


\section{ECG E FREQUÊNCIA CARDÍACA}

A frequência cardíaca indica a quantidade de vezes que o coração bate por minuto (BPM). Em adultos varia entre $60 \mathrm{e}$ 100 BPM. Porém, ela varia com a idade, se a pessoa faz alguma atividade física ou se possui alguma doença cardíaca.

Basicamente, quanto mais força o coração precisa fazer para mandar o sangue para o corpo, maior será seu esforço e consequentemente, maior será a frequência cardíaca dessa pessoa. Por isso, existe uma taxa ideal que varia conforme a idade.

- Até 2 anos de idade: 120 a 140 BPM;

- Entre 8 anos até 17 anos: 80 a 100 BPM;

- Adulto sedentário: 70 a 80 BPM;

- Adulto que faz atividade física e idosos: 50 a 60 BPM.

O cálculo da frequência neste trabalho é data pela equação 4:

$$
F C=\frac{60 \cdot T A}{R 2-R 1}
$$

Onde:

$F C$ é a Frequência cardíaca;

$T A$ é a taxa de amostragem;

$R 2-R 1$ é a diferença amostral de dois picos $\mathrm{R}$ na série temporal

O eletrocardiograma pode ser usado para detectar ou acompanhar vários problemas cardíacos, dentre alguns deles temos:

- Irregularidades no ritmo cardíaco (arritmia), seja por um coração acelerado (taquicardia), devagar (bradicardia) ou fora do ritmo;

- Artérias bloqueadas ou estreitas no coração (doença arterial coronariana);

- Doenças que isolam o coração (derrame pericárdico ou pneumotórax);

- Inflamação da membrana que envolve o coração (pericardite);

- Doença de Chagas;

- Hipertrofia das câmaras cardíacas (átrios e ventrículos).

\section{RESULTADOS}

A Figura 4 mostra o ECG original, sem nenhum tratamento. 17 mil amostras foram coletadas a uma taxa de amostragem de $1 \mathrm{KHz}$.

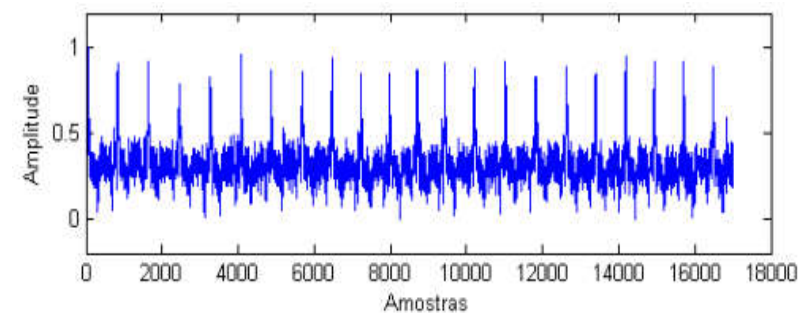

Fig. 4 ECG original: Amostra 1.
A seguir, será mostrado todos os resultados obtidos. A Figura 5 apresenta o sinal do ECG após a filtragem das componentes da baixa frequências (FFT), e eliminação dos 85 valores iniciais e finais do sinal original.

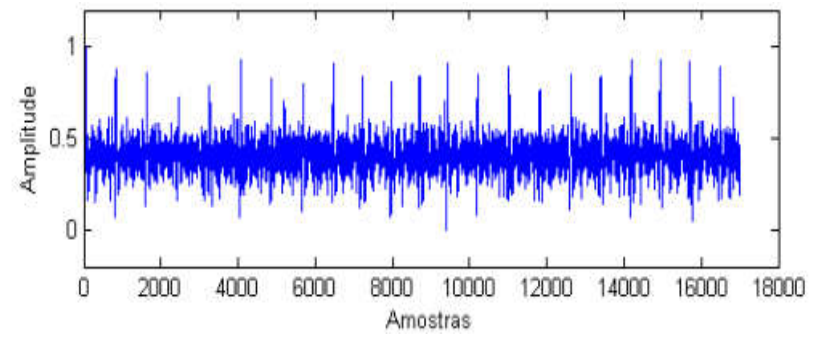

Fig. 5 Sinal filtrado pela FFT.

Após o primeiro filtro por limiar, pode-se obter os primeiros valores de picos R do ECG, conforme Figura 6.

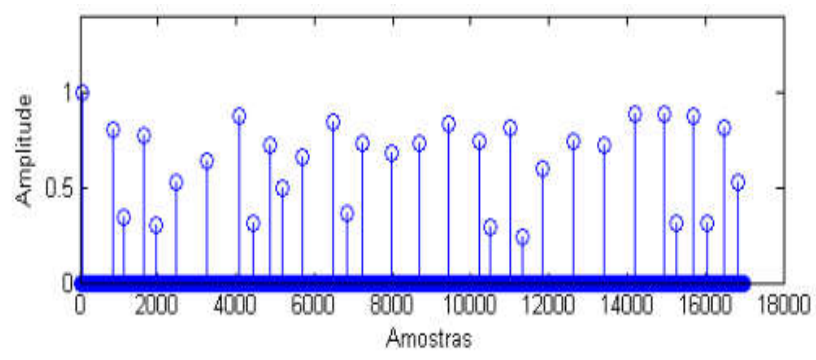

Fig. 6 Primeiro filtro por limiar.

Claramente há picos que podem ser ou não picos $\mathrm{R}$, tais picos podem ser identificados facilmente observando a escala no eixo $\mathrm{Y}$, valores menores de 0,5 provavelmente não se tratam da onda de interesse. Portanto, aplicando a condição de limiar novamente para que estes pontos sejam classificados ou não, $o$ filtro deve ser utilizado pela segunda vez, mas com um incremento da função ecgdemomaxmin, diminuindo a taxa de limiar e restringindo ainda mais a diferença entre os picos. A Figura 7 apresenta os novos picos detectados.

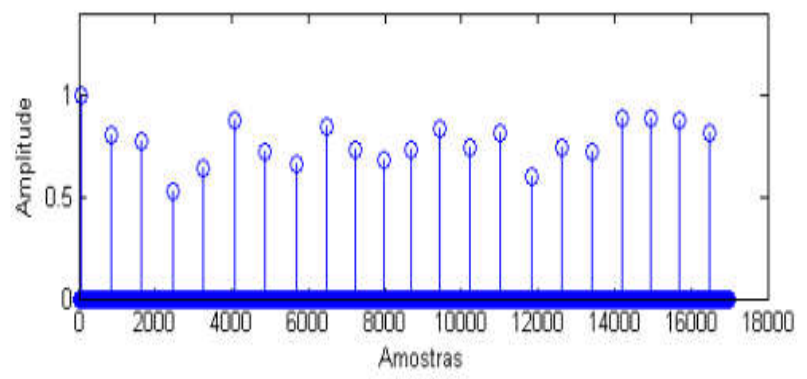

Fig. 7 Segundo filtro por limiar.

O segundo filtro conseguiu eliminar muitos valores que não seriam significativos dentro dos padrões dos ECG, e preservou os valores que são importantes para se obter mais precisamente a frequência cardíaca. Normalizando estes valores de acordo com a Figura 8, tem-se o resultado final do pico R encontrado no sinal do ECG em questão. Os pontos de pico da onda R são destacados onde realmente eles acontecem. O Sinal do ECG é plotado juntamente com o sinal filtrado pela segunda vez. Um comparativo é mostrado na Figura 9. 


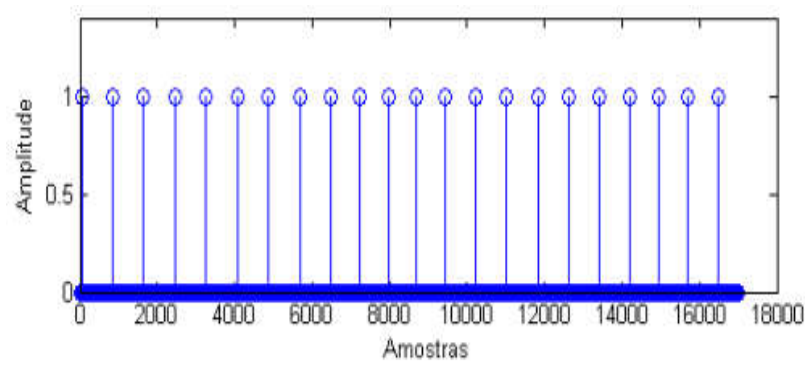

Fig. 8 Resultado final do filtro por limiar.

O sinal do ECG original é plotado em tempo contínuo e o sinal filtrado em tempo discreto, o que possibilita o cálculo da frequência cardíaca de acordo com a equação 4.

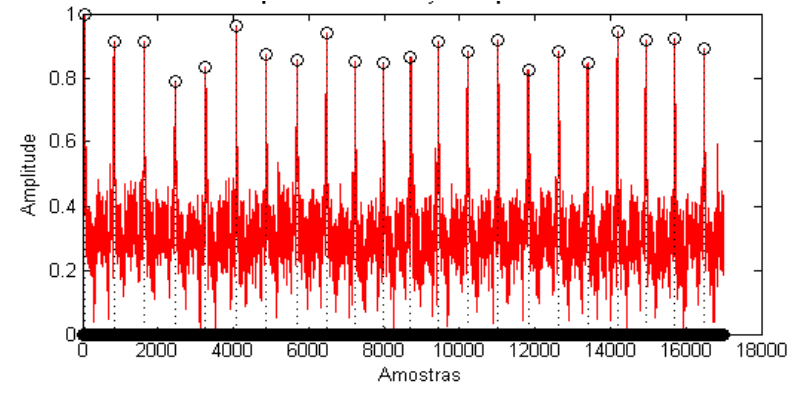

Fig. 9 Picos identificados no sinal original do ECG.

Diante de todos os resultados encontrados anteriormente, é possível obter os valores da frequência cardíaca desse ECG. A Figura 10 mostra os valores encontrados ponto a ponto, ou seja, para cada par de pico $\mathrm{R}$ identificado e classificado no processo realizado. Pela Figura 10 ainda é possível obter a frequência cardíaca média, usando no denominador da equação 4, o primeiro pico R identificado e o último. $\mathrm{O}$ Valor encontrado pela média é de 80,4 BPM.

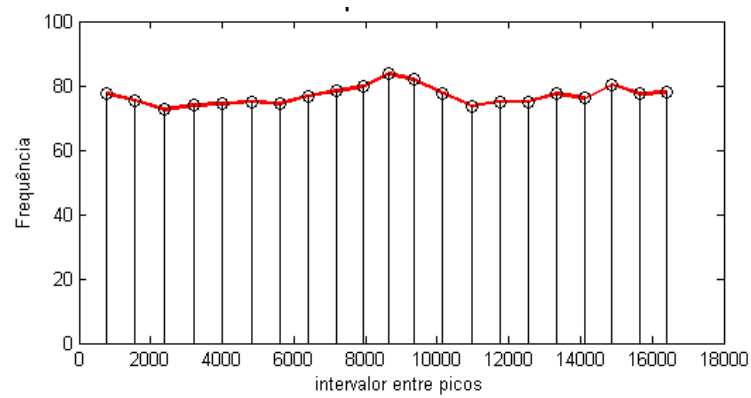

Fig. 10 Frequência cardíaca do ECG após filtragem em duas etapas.

Os próximos resultados obtidos são referentes a Transformada de Wavelet Discreta. A Figura 11 apresenta o sinal de ECG de outra pessoa, sem nenhum filtro ou tratamento, onde a amostra tem tamanho igual a 7200 e foi obtida com uma taxa de amostragem de $1 \mathrm{KHz}$.

Antes de aplicar a TWD, o sinal foi suavizado pela função smooth do Matlab. Essa função suaviza os dados no vetor usando um filtro de média móvel, e o período padrão para a média móvel é 5. A Figura 12 mostra o sinal do ECG da segunda pessoa suavizado.

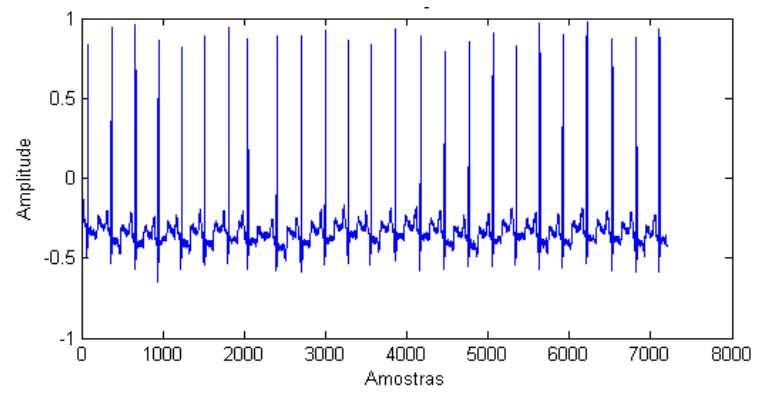

Fig. 11 ECG original: Amostra 2.

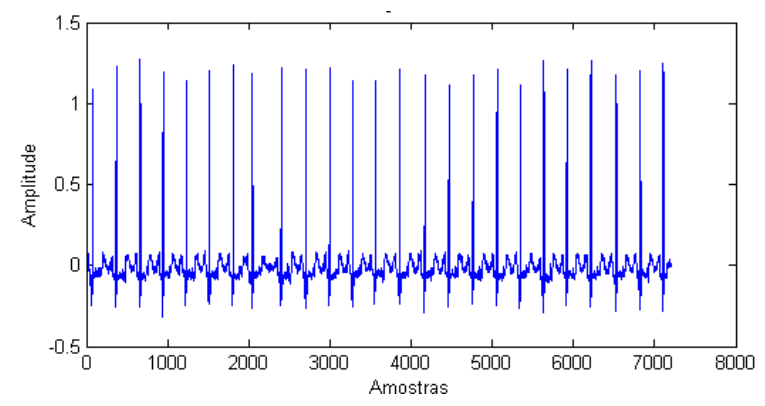

Fig. 12 Sinal original suavizado.

Fazendo uma análise dos dados obtidos após a aplicação da TWD, verificou-se que os níveis d4 e d5 são mais viáveis para distinguir a onda R. Em virtude disto e pelo fato do nível d4 apresentar amplitudes inferiores, foi escolhido o nível d5 para efetuar a detecção do pico R, e esse nível é mostrado na Figura 13.

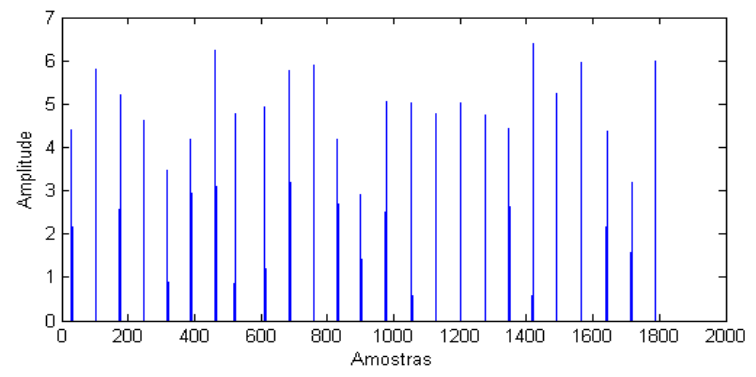

Fig. 13 Banda de frequência d5 da TWD: ECG amostra 2.

Com os mais satisfatórios picos de onda $\mathrm{R}$ encontrados, segue o mesmo procedimento para cálculo da frequência cardíaca realizado na primeira pessoa, no filtro por limiar. A equação 4 é usada novamente e o resultado apresentado na Figura 14.

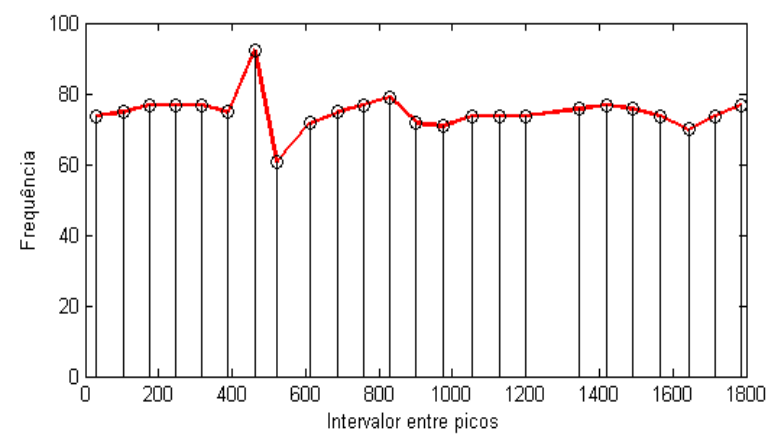

Fig. 14 Frequência cardíaca após aplicação da TWD. 
Utilizando do mesmo conceito referente a Figura 10 para o cálculo da frequência cardíaca média, ou seja, o primeiro menos último valor do pico da onda $\mathrm{R}$ da Figura 14, dividido pelo número de picos encontrados, é o denominador da equação 4. O numerador não é alterado, pois, a taxa de amostragem foi a mesma, $1 \mathrm{KHz}$. Portanto a frequência cardíaca média para o ECG da segunda pessoa é 74,5 BPM.

\section{CONCLUSÃO}

Neste trabalho são comparadas duas metodologias de detecção da onda R do ECG. As abordagens combinam a técnica do limiar, sendo: uma puramente por limiar e outra com limiar na Transformada de Wavelet Discreta.

As duas técnicas apresentam resultados satisfatórios, mas a TWD demanda menos etapas para detectar os picos. Isso interfere no custo computacional e na agilidade para se obter o resultado final, que pode ser bem relevante quando se trata de condições clínicas.

O cálculo da frequência cardíaca ponto a ponto possibilita uma leitura mais detalhada da mesma. Enquanto que a frequência cardíaca média não apresenta detalhes no decorrer do ciclo cardíaco, e sim apenas um valor final.

Os filtros possibilitaram a eliminação de artefatos antes considerados ondas de interesse (onda $\mathrm{R}$ ), o que levava há uma leitura menos precisa.

Em trabalhos futuros, os mesmos algoritmos podem ser aplicados afim de eliminar artefatos do eletroencefalograma e do eletromiograma para identificação de espículas epileptiformes e/ou potenciais de ação de unidade motora, apenas mudando os limiares e no caso da TWD, as bandas de frequência. Ainda pode-se considerar mais filtros em frequências específicas, dependo do sinal em estudo. A abrangência do algoritmo pode também ser estendida e levada a meios industriais, como por exemplo, na manutenção preditiva de motores de indução, em que separar e classificar as frequências presentes nos sinais elétricos podem identificar futuras falhas.

\section{AGRADECIMENTOS}

Agradecemos a Universidade Federal de São João del-Rei e a Fundação de Amparo à Pesquisa do Estado de Minas Gerais FAPEMIG pelo apoio financeiro.

\section{REFERÊNCIAS}

Friesen, G. M., Jannett, T. C., Jadallah, M. A., Yates, S. L., Quint, S. R., \& Nagle, H. T. (1990). A comparison of the noise sensitivity of nine QRS detection algorithms. IEEE Transactions on biomedical engineering, 37(1), 85-98.

Gopalakrishnan Nair, T. R., Geetha, A. P., \& Asharani, M. (2013). Continuous digital ECG analysis over accurate Rpeak detection using adaptive wavelet technique. Journal of medical engineering \& technology, 37(7), 429-435.

Junior, E. A., de Medeiros Valentim, R. A., \& Brandao, G. B. (2016). Real time QRS detection based on redundant discrete wavelet transform. IEEE Latin America Transactions, 14(4), 1662-1668.
Kohler, B. U., Hennig, C., \& Orglmeister, R. (2002). The principles of software QRS detection. IEEE Engineering in Medicine and Biology Magazine, 21(1), 42-57.

Madeiro, J. P. V., Cortez, P. C., Marques, J. A. L. (2009). Análise comparativa de desempenho das transformadas Wavelet e Hilbert na detecção do QRS e ECG. Revista Brasileira de Engenharia Biomédica, (25), 153-166.

Merah, M., Abdelmalik, T. A., \& Larbi, B. H. (2015). R-peaks detection based on stationary wavelet transform. Computer methods and programs in biomedicine, 121(3), 149-160.

Silva, G.M.A., Soares, C. P., Sotomayor, O. A. Z. (2015). Teoria Wavelet na análise de sinais de ECG como ferramentas de auxílio na detecção de arritmias cardíacas. XVI Simpósio Brasileiro de automação Inteligente (SBAI), Natal - RN, Brasil.

Thakor, N. V., Webster, J. G., \& Tompkins, W. J. (1984). Estimation of QRS complex power spectra for design of a QRS filter. IEEE Transactions on biomedical engineering, (11), 702-706. 